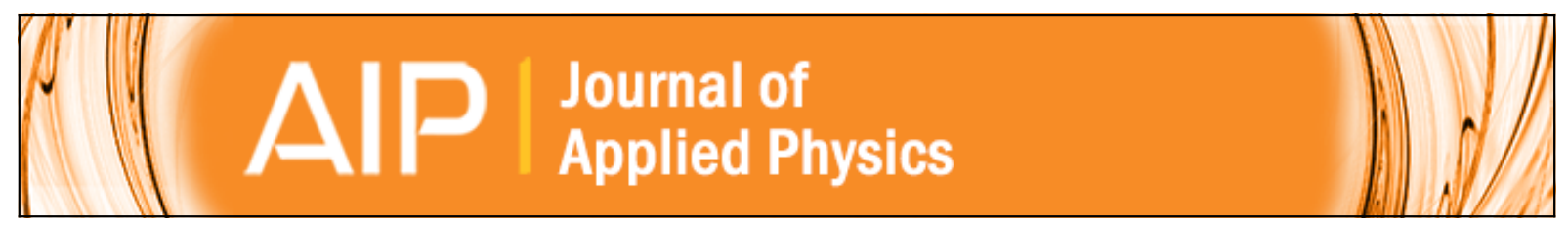

\title{
Computational analysis of ionic polymer cluster energetics
}

Lisa Mauck Weiland and Donald J. Leo

Citation: Journal of Applied Physics 97, 013541 (2005); doi: 10.1063/1.1819974

View online: http://dx.doi.org/10.1063/1.1819974

View Table of Contents: http://scitation.aip.org/content/aip/journal/jap/97/1?ver=pdfcov

Published by the AIP Publishing

\section{Articles you may be interested in}

Highly enhanced interfacial adhesion properties of steel-polymer composites by dot-shaped surface patterning J. Appl. Phys. 109, 074302 (2011); 10.1063/1.3567113

Ionic polymer cluster energetics: Computational analysis of pendant chain stiffness and charge imbalance J. Appl. Phys. 97, 123530 (2005); 10.1063/1.1937475

Micromechanics of actuation of ionic polymer-metal composites

J. Appl. Phys. 92, 2899 (2002); 10.1063/1.1495888

Erratum: "Surface-rheological measurements on glass forming polymers based on the surface tension driven decay of imprinted corrugation gratings" [J. Chem. Phys. 112, 4262 (2000)]

J. Chem. Phys. 114, 9685 (2001); 10.1063/1.1352732

Surface-rheological measurements on glass forming polymers based on the surface tension driven decay of imprinted corrugation gratings

J. Chem. Phys. 112, 4262 (2000); 10.1063/1.481002

MIT LINCOLN LABORATORY CAREERS

Discover the satisfaction of innovation and service to the nation
- Space Control

- Air \& Missile Defense

- Communications Systems \& Cyber Security

- Intelligence, Surveillance and

Reconnaissance Systems
- Advanced Electronics

- Tactical Systems

- Homeland

Protection

- Air Traffic Control

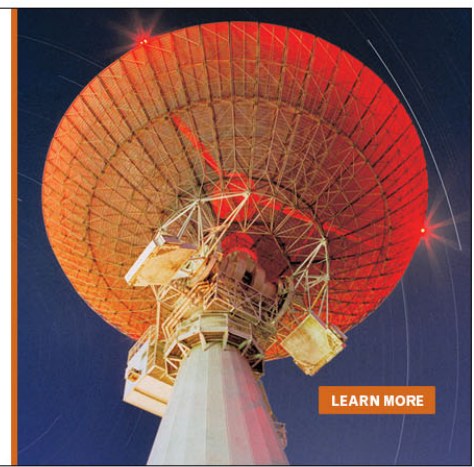




\title{
Computational analysis of ionic polymer cluster energetics
}

\author{
Lisa Mauck Weiland ${ }^{\text {a) }}$ and Donald J. Leo \\ Center for Intelligent Material Systems and Structures, Mechanical Engineering Department, Virginia Tech, \\ Blacksburg, Virginia 24061-0261
}

(Received 6 July 2004; accepted 24 September 2004; published online 16 December 2004)

\begin{abstract}
In recent years there has been considerable study of the potential mechanisms underlying the electromechanical response of ionic-polymer-metal composites. The most recent models have been based on the response of the ion-containing clusters that are formed when the material is synthesized. Most of these efforts have employed assumptions of uniform ion distribution within spherical cluster shapes. This work investigates the impact of dispensing with these assumptions in order to better understand the parameters that impact cluster shape, size, and ion transport potential. A computational micromechanics model has been developed to predict the equilibrium state of a single cluster of an ionomeric polymer with cluster morphology. No assumptions are made regarding the distribution of charge or the shape of the cluster. For a constant solvated state, the model tracks the position of individual ions within a given cluster in response to ion-ion interaction, mechanical stiffness of the pendant chain, cluster surface energy, and external electric-field loading. Expressions are developed to directly account for forces imposed on ions due to ion-cluster surface interaction. Results suggest that ion pairing is rarely complete; this in turn suggests that the classic assumptions will tend to underpredict electromechanical actuation response. (C) 2005 American Institute of Physics. [DOI: 10.1063/1.1819974]
\end{abstract}

\section{INTRODUCTION}

It has been over a decade since the transduction properties of ionic-polymer-metal composites (IPMCs) were observed. ${ }^{1-5}$ In that time there has been considerable conjecture over the potential applications of these soft transducers $2,3,6,7$ and considerable fruitful investigation towards these ends. ${ }^{8-10}$ However, many aspects of ionomer electromechanical behavior still are not understood, impeding full exploitation of these devices. For instance, because these devices (for most polymer and associated counterion forms) display a fast response followed by a slow "relaxation" response when fully solvated, it is clear that at least two different electromechanical response mechanisms are stimulated by the application of an electric field. The nature of these mechanisms is still under investigation.

Most models of the transduction behavior rely on the cluster morphology of the ionic polymer layer as proposed by Hsu and Gierke, ${ }^{11}$ where the material has been solvated with water. In brief, the backbone of the ionic polymer chain is hydrophobic, while the side chains terminate in hydrophilic ionic groups. Hsu and Geirke propose that these hydrophilic ionic side groups and the water that has been taken up by the material cluster together. The model further suggests an idealized structuring whereby the clusters are of essentially constant radius, uniformly distributed throughout the material, and interconnected by channels. Subsequently, many have sought to quantify the cluster radius and spacing as functions of polymer equivalent weight, hydration level, counterion, etc. See for instance the modeling works of Datye and co-workers ${ }^{12,13}$ and the experimental work of Lehmani et al. ${ }^{14}$

${ }^{\mathrm{a})}$ Electronic mail: mauck-weiland@vt.edu
Early transduction models proposed an entirely hydraulic description of the electromechanical response. In this approach counterions and water migrate through the thickness of the material in response to electric field; this in turn results in differential swelling of the clusters and the observed bending response. Because these models are consistent with a substantial proportion of the experimentally observed response, considerable strides have been made in the effort to develop them. ${ }^{15}$ However, it has been noted that these models are in conflict with certain aspects of recently obtained experimental observations. ${ }^{16}$ A subsequent hybrid model proposed by $\mathrm{Li}$ and Nemat-Nasser ${ }^{17,18}$ addresses the abovenoted multimechanisms by considering the interplay of multiple contributions to the overall energy state including elastic, electrostatic, and osmotic effects. In the process of developing their models they also assume uniform distribution of the ionic side groups over the surface of each spherical cluster. Further, the formation of pseudodipoles, or ion pairing, is assumed when counterions are present; pairing is assumed to be complete and uniformly, radially oriented.

Based on the results of a preliminary model, ${ }^{19}$ the model presented in this work directly addresses the impact of releasing the assumption of uniform and balanced ion distribution through application of a computational approach. The current work also introduces explicit consideration of the effect of cluster surface energy in relation to ion-cluster surface interaction and allows for predictions of nonspherical cluster shapes. Ultimately, this model identifies the lowenergy state of a cluster for specified distributions of ions, and thus no transport of ions outside the cluster is considered. Rather, transport in this study is considered indirectly via the state of the cluster. The intent of this work is to 


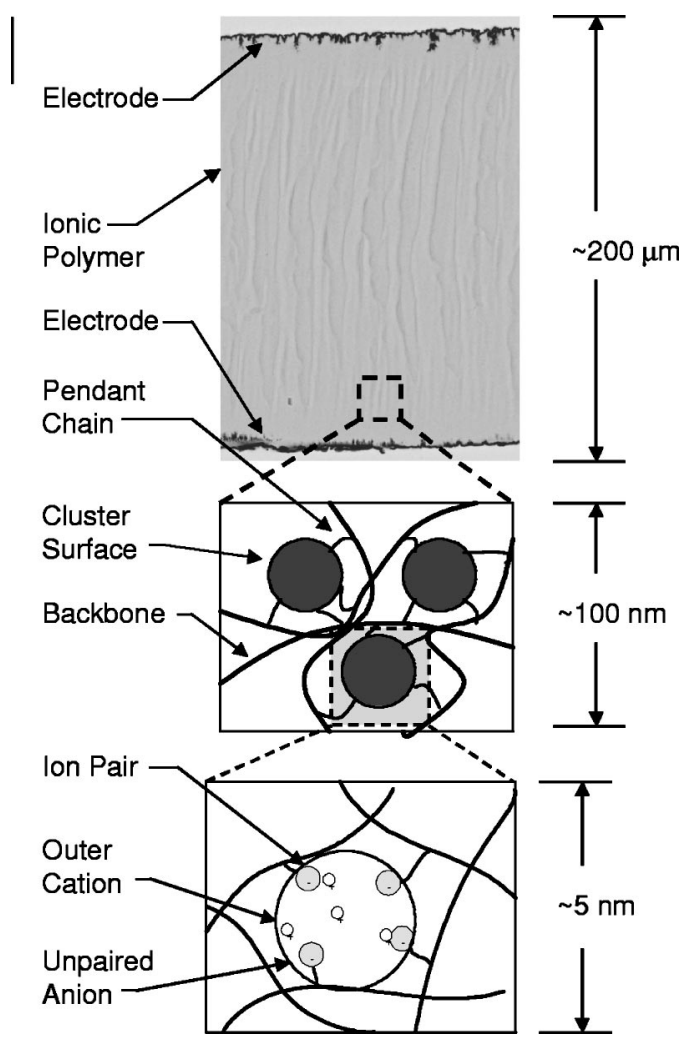

FIG. 1. Identification of the region of study (a single cluster) as it relates to an IPMC.

facilitate the application of the larger length scale models, such as those noted above, through identification of sources of error and their impact.

The paper is organized as follows: Section II discusses the base assumptions and theoretical development. Section III addresses construction of the model. Section IV fits the model to a specific ionic polymer case, which serves as the base line from which trends may be observed. Section V presents model predictions. Section VI provides a discussion. Section VII concludes the work.

\section{MODEL DEVELOPMENT}

Our goal is to understand the interactions of ions within a cluster as a function of relevant polymer properties. To this end we base our analysis on the cluster model shown in Fig. 1. The cluster model consists of a specified number of negatively charged ionic groups ("anions") and an equal number of positively charged ions ("cations"). We assume that the cluster is solvated with a liquid and that this liquid creates a surface tension at the boundaries of the cluster which will restrict the motion of cations and anions. Further, we assume that this cluster surface or hydrophobic-hydrophilic boundary, is defined by the location of the anions covalently bonded to polymer side chains.

We define ion pairing as the close proximity of a cation and an anion. Unlike some previous models which have assumed that ion pairing is complete, the present model allows the existence of "unpaired ions," i.e., those cations that are not in close proximity to an anion. Further, the existence of cations located outside the anion boundary shell, termed "outer cations," is hypothesized.

The model is constructed such that the ions are allowed to move independently in a manner governed by the summation of electrostatic, elastic, and cluster surface forces, where this summation dictates the proposed stepping direction. The summation of the corresponding energy terms is used to establish the admissibility of a force-driven step toward a lower-energy state. In both summations, it is assumed that there is no direct coupling among the critical terms. These summations are given by

$$
\begin{aligned}
& \mathbf{F}_{\text {tot }}=\mathbf{F}_{\text {ext }}+\mathbf{F}_{\text {ion-ion }}+\mathbf{F}_{\mathrm{pc}}+\mathbf{F}_{s}, \\
& U_{\text {tot }}=U_{\text {ext }}+U_{\text {ion-ion }}+U_{\mathrm{pc}}+U_{s},
\end{aligned}
$$

where the subscript "ext" refers to externally applied electric field, "ion-ion" refers to electrostatic ion-ion interactions, pc refers to pendant chain response to motion of the attached anion, and $s$ refers to the interaction of ions with the cluster surface. In the application of Eq. (1), each ion is considered individually in order to determine the direction of stepping. Equation (2) is applied to establish the admissibility of the step direction via minimization of energy. Convergence (equilibrium) of the system as a whole is reached when all ion steps are rejected as inadmissible, thus the total system energy has been minimized for the given step size. In the following discussion, each of the terms in Eqs. (1) and (2) are addressed individually.

\section{A. Externally applied electric field}

The only external electric-field loading considered is the electric field applied globally via the IPMC electrodes. The force exerted on any given ion due to the total external electric field is found by multiplying the charge of that ion, $c$, by the externally applied field

$$
\mathbf{F}_{\mathrm{ext}}=c \mathbf{E}_{\mathrm{ext}} .
$$

Ion position is monitored by a position vector, $\mathbf{p}$, which is updated after each step. For uniform and constant $\mathbf{F}_{\text {ext }}$, the potential energy associated with ion position due to external fields is given by

$$
U_{\mathrm{ext}}=-\mathbf{F}_{\mathrm{ext}} \cdot \mathbf{p} .
$$

Because only changes in this value are of interest, the constant of integration has been omitted.

\section{B. Ion-ion interaction}

The force on any given ion due to every other ion is expressed by multiplying the electric field by the charge of the ion being considered,

$$
\mathbf{F}_{\text {ion-ion }}=c \mathbf{E}_{\text {ion-ion }} .
$$

$\mathbf{E}_{\text {ion-ion }}$ is given by 


$$
\begin{aligned}
\mathbf{E}_{\text {ion-ion }} & =\sum_{j=1, j \neq i}^{\left(n_{\text {an }}+n_{\text {cat }}\right)} E_{i-j} \\
& =\sum_{j=1, j \neq i}^{\left(n_{\text {an }}+n_{\text {cat }}\right)} \frac{9 \times 10^{9} c_{j}}{\varepsilon \cdot r_{i-j}^{3}}\left[1-\left(\frac{r_{0}}{r_{i-j}}\right)^{10}\right] \cdot \mathbf{r}_{i-j},
\end{aligned}
$$

where $\mathbf{E}_{i-j}$ is the electric field acting on ion $i$ due to ion $j, n_{\text {an }}$ is the total number of anions, $n_{\text {cat }}$ is the total number of cations, $\varepsilon$ is the net effective dielectric constant within the cluster, $c_{j}$ is the charge of the $j$ th ion, $r_{o}$ is the equilibrium separation between atoms, and $\mathbf{r}_{i-j}$, given by

$$
\mathbf{r}_{i-j}=\mathbf{p}^{i}-\mathbf{p}^{j},
$$

is the vector distance separating ion $i$ from ion $j$, while $r_{i-j}$ is the magnitude of $\mathbf{r}_{i-j}$. In Eq. (6), $r_{i-j}$ is raised to the power of 3 instead of the power of 2 because $\mathbf{r}_{i-j}$ must be normalized. The first parenthetical term of Eq. (6) is consistent with classic electrostatic theory. ${ }^{20}$ The second parenthetical term derives from the nature of an ionic bond where Pauli repulsion prevents overlapping ion clouds and the exponential value is found experimentally to vary between 3 and $12 .^{21,22}$ In the absence of available experimental data for the case studied in this effort, a relatively large exponent from within this range is selected in order to minimize long-range effects. By applying this expression, cation-anion pairs necessarily respond in a manner consistent with dipole response without having to explicitly account for the pairing.

Based on Eqs. (6) and (7), the force acting on ion $i$ is a function of position, $\mathbf{p}^{i}$, and thus, the change in potential energy associated with incrementally moving the ion must also be a function of position, such that,

$$
F_{\text {ion-ion }}=-\nabla U_{\text {ion-ion }},
$$

where the gradient is taken in $r$, resulting in

$$
\begin{aligned}
U_{\text {ion-ion }} & =\sum_{j=1, j \neq 1}^{\left(n_{\text {an }}+n_{\text {cat }}\right)} U_{i-j} \\
& =\sum_{j=1, j \neq 1}^{\left(n_{\text {an }}+n_{\text {cat }}\right)} \frac{9 \times 10^{9} c_{j} c_{i}}{\varepsilon}\left(\frac{1}{r_{i-j}}-\frac{1}{11 r_{i-j}}\left(\frac{r_{0}}{r_{i-j}}\right)^{10}\right),
\end{aligned}
$$

where $U_{\text {ion-ion }}$ is naturally path independent.

\section{Pendant chain response}

Any motion of the anion is necessarily resisted/assisted by the deformation of the pendant chain to which it is covalently bonded. This resistance/assistance is approximated by a linear reversible spring, such that,

$$
\mathbf{F}_{\mathrm{pc}}=k_{\mathrm{pc}} \mathbf{x}=-k_{\mathrm{pc}}\left(\mathbf{p}-\mathbf{p}_{0}\right),
$$

where $\mathbf{p}_{o}$ is the initialized anion position and $k_{p c}$ is estimated from the models of Datye et al. ${ }^{12,13}$ as

$$
k_{\mathrm{pc}} \approx \frac{3 k_{B} T}{2\left\langle h^{2}\right\rangle},
$$

where $k_{B}$ is Boltzmann's constant, $T$ is absolute temperature, and $\left\langle h^{2}\right\rangle$ is the mean-square end-to-end distance between ionic groups. Based on the values provided by Datye et al.,

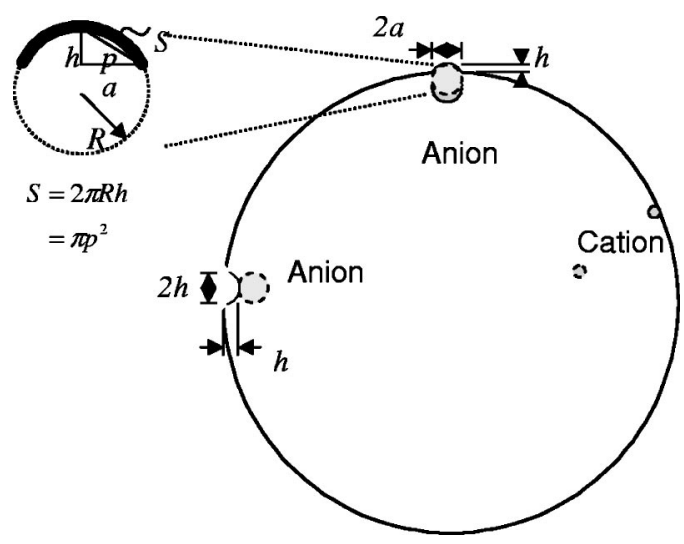

FIG. 2. Outward cation movement has the same effect as outward anion movement. Inward cation movement has no cluster surface effect.

this value can be calculated for the cases of 8 and 20 dipoles at room temperature, while linear extrapolation is used to estimate the value for other numbers of ion pairs.

For the case of constant $k_{\mathrm{pc}}$, the change in energy associated with anion displacement from position $\mathbf{p}_{0}$ to position $\mathbf{p}$ is given by

$$
U_{\mathrm{pc}}=\frac{1}{2} k_{p c}\left(\mathbf{p}-\mathbf{p}_{0}\right) \cdot\left(\mathbf{p}-\mathbf{p}_{0}\right) .
$$

\section{Cluster surface-ion interaction}

In the absence of all external stimuli (gravity, ion motion, etc.), the cluster shape minimizing cluster surface energy is spherical. Most ionic polymer models assume spherical cluster shapes, as proposed by Hsu and Gierke. ${ }^{11}$ However, cluster surface-ion interaction can distort this shape away from this spherical, low-energy state. In the development of the appropriate expressions to be substituted into Eqs. (1) and (2), it is best to begin with the energy expression from which the force expression is developed.

The expression for surface energy is commonly given by

$$
U_{s}=\gamma A \text {, }
$$

where $\gamma$ is the constant surface-tension coefficient and $A$ is the area of the surface. For a spherical cluster, the total surface energy is given by $U_{S \text {, sphere }}=\gamma\left(4 \pi r^{2}\right)$.

Cluster surface response to ion interaction is local. Therefore, only changes in the local area are considered. It is assumed that anions are always in contact with the cluster surface, while cations are not. Thus, if an anion moves either inward or outward from its initialized position, the surface will resist the motion. A cation may move to the interior of the cluster with no surface energy penalty (refer to Fig. 2). Because both cation and anion radii are much smaller than the cluster radius, the local region impacted by ion-surface interaction may be approximated by a flat surface, resulting in a surface response which is invariant to ion size. (It can be shown that the maximum error introduced by this approximation is $5 \%$, which occurs at hypothetical large displacements of an anion, which in turn are rarely predicted by the model.) 
TABLE I. Input parameters, symbols, and values.

\begin{tabular}{lcc}
\hline \hline \multicolumn{1}{c}{ Parameter } & Symbol & Value \\
\hline Cluster radius & $r_{\mathrm{cl}}$ & $2.105 \mathrm{~nm}$ \\
Ion pair separation & $r_{\max }$ & $0.361 \mathrm{~nm}$ \\
Number of anions & $n_{\mathrm{an}}$ & 26 \\
Anion radius & $r_{\mathrm{an}}$ & $0.24 \mathrm{~nm}$ \\
Ion charge & $c$ & $\pm 1.602 \times 10^{-19} \mathrm{C}$ \\
Number of cations & $n_{\mathrm{cat}}$ & 26 \\
Cation radius (Na $\left.{ }^{+}\right)$ & $r_{\mathrm{cat}}$ & $0.097 \mathrm{~nm}$ \\
Pendant chain stiffness & $k_{p c}$ & $0.0125 \mathrm{~N} / \mathrm{m}$ \\
Effective dielectric constant & $\varepsilon$ & 78 \\
Surface energy constant & $\gamma$ & $0.0729 \mathrm{~N} / \mathrm{m}$ \\
Perturbation range & $\alpha_{\text {per }}$ & $0^{\circ}, 30^{\circ}, 45^{\circ}$ \\
Maximum step size & & $0.002 \mathrm{~nm}$ \\
External electric field & $\mathbf{E}_{a}$ & $1 \times 10^{4} \mathbf{k} \mathrm{V} / \mathrm{m}$ \\
\hline \hline
\end{tabular}

Consider first the case of an anion moving outward from its initialized position by step, $h$. Referring to Fig. 2, the surface of the ion which has perturbed the cluster is given by

$$
A_{2}=2 \pi r_{\text {ion }} h,
$$

while the original area of this region is given by

$$
A_{1} \approx \pi a^{2}=\pi\left(2 r_{\text {ion }} h-h^{2}\right) .
$$

Equation (15) is derived from the mensuration formula for the surface area of a sphere zone. Next, consider that for the current case, interest is in variations away from the lowenergy, initialized state. Change in the surface energy due to change in the local surface area is given by

$$
\Delta U_{s}=\gamma \Delta A=\gamma\left[2 \pi r_{\text {ion }} h-\pi\left(2 r_{\text {ion }} h-h^{2}\right)\right] .
$$

Simplifying the above equation and recognizing that $h$ is the magnitude of the step beyond the initialized position at the cluster surface,

$$
h=|\mathbf{p}|-r_{\mathrm{cl}}+r_{\text {ion }},
$$

the expression for the change in cluster surface energy due to the local disturbance may be expressed as

$$
\Delta U_{s}=\gamma \pi h^{2}=\gamma \pi\left[|\mathbf{p}|-r_{\mathrm{cl}}\right]^{2} .
$$

Finally, an expression for the force imposed on the ion due to the distortion may be developed, where

$$
\mathbf{F}_{s}=-\nabla\left(\Delta U_{s}\right)=-2 \gamma \pi\left[|\mathbf{p}|-r_{\mathrm{cl}]}\right] \frac{\mathbf{p}}{|\mathbf{p}|} .
$$

The radius of the ion drops out of both the energy and force expressions. Thus, while this discussion began by identifying an outward motion of an anion, Eqs. (18) and (19) are valid for both anion and cation outward motion with the caveat that these expressions are not to be employed at all if a cation is at a position interior to the cluster (or $|\mathbf{p}|<r_{\mathrm{cl}}$ ). Furthermore, these expressions are valid for both large and small distortions.

It can be shown that an inward anion step results in the analogous expressions for force and energy change, even if the deformation shape is set to a semispherical shape of radius $h$. The resulting final expressions are given by

$$
\Delta U_{s}=\gamma \pi\left[r_{\mathrm{cl}}-|\mathbf{p}|\right]^{2}
$$

and

$$
\mathbf{F}_{s}=2 \gamma \pi\left[r_{\mathrm{cl}}-|\mathbf{p}|\right] \frac{\mathbf{p}}{|\mathbf{p}|} .
$$

Lastly, it can be shown that the above force expressions, when converted to an expression for the differential pressure between the interior and exterior of the cluster (by dividing by the local area being considered), are equivalent to Laplace's formula, ${ }^{23}$

$$
P=\gamma\left(\frac{1}{r_{\alpha}}+\frac{1}{r_{\beta}}\right) \text {. }
$$

\section{MODEL CONSTRUCTION}

The model input values are based on those typically applied for the case of Nafion ${ }^{\circledR}$ (DuPont) fully solvated with water and with sodium as the cation (Table I). The majority of the values assigned are based on Datye et al. ${ }^{12}$ The exceptions are the values for the dielectric constant and surface energy constant, where these are based on typical values for water. ${ }^{24-26}$ Application of these macroscopically obtained, uniform values represent a simplifying assumption.

Using the input value for cluster radius, spherical coordinates are used to initially, uniformly distribute the anions in three-dimensional space on the surface of the cluster. If the perturbation range, $\alpha_{\text {per }}$, is nonzero, then each of the anions is perturbed from its initial position in terms of $\theta$ and $\phi$ within the range of $\pm \alpha_{\mathrm{per}} / 2$. Perturbations are accomplished by calling a random number, $n$, between -0.5 and +0.5 , and applying it such that

$$
\Theta_{\text {new }}=\Theta_{\text {initial }}+n \alpha_{\text {per }} .
$$

Similarly, another random number is called to perturb the value for $\phi$. This process is repeated for each anion. Following the distribution of the anions, the cations are randomly placed within the cluster bounds, where overlapping placements are rejected.

From this starting point, the ions are stepped toward their equilibrium positions as previously described. When 
TABLE II. Variations in predicted cluster response due to step size (averages over 10 random distributions).

\begin{tabular}{|c|c|c|c|c|c|c|c|}
\hline $\begin{array}{l}\text { Initial } \\
\text { step }\end{array}$ & $\begin{array}{c}\text { Iterations } \\
\text { (initial) }\end{array}$ & $\begin{array}{c}\text { Ion } \\
\text { pairs }\end{array}$ & $\begin{array}{c}\Delta \text { En } 1 \\
\times 10^{-19} \\
(\mathrm{~J})\end{array}$ & $\begin{array}{c}\text { Step } \\
\text { decrease } \\
\text { factor }\end{array}$ & $\begin{array}{c}\text { Iterations } \\
\left(E_{\text {applied }}\right)\end{array}$ & $\begin{array}{c}\text { Ion } \\
\text { pairs }\end{array}$ & $\begin{array}{c}\Delta \mathrm{En} 2 \\
\times 10^{-19} \\
(\mathrm{~J})\end{array}$ \\
\hline $2 r_{\text {cat }}$ & 11.0 & 19.8 & -3.57 & 2.3 & 4.8 & 21.1 & -3.65 \\
\hline$r_{\text {cat }}$ & 21.0 & 21.2 & -3.63 & 2.7 & 22.7 & 22 & -3.73 \\
\hline$r_{\text {cat }} / 10$ & 235.0 & 22.6 & -3.85 & 12.7 & 1751.3 & 22.6 & -3.92 \\
\hline$r_{\mathrm{cat}} / 30$ & 749.9 & 19.7 & -3.90 & 38.8 & 5707.8 & 19.7 & -3.91 \\
\hline$r_{\text {cat }} / 40$ & 1063.6 & 19.1 & -3.91 & 55.7 & 4030.6 & 19.1 & -3.91 \\
\hline$r_{\mathrm{cat}} / 50$ & 1289.9 & 18.9 & -3.91 & 69.4 & 998.8 & 18.9 & -3.91 \\
\hline$r_{\text {cat }} / 100$ & 2439.1 & 18.4 & -3.91 & 136.8 & 44 & 18.4 & -3.91 \\
\hline
\end{tabular}

this process begins, the external electric field is zero while the ions find equilibrium positions based on their initialized distribution ("Equilibrium 1"). From the calculated force, a step in the same direction and of maximum magnitude $0.002 \mathrm{~nm}$ is tested (selection of this step size is discussed in the following section). If the step leads to ion overlap, the step size is reduced or rejected depending on the proximity of the conflicting ion. The step is then tested to confirm that it leads to a lower-energy state via Eq. (2); if it fails this test, the step is rejected. This method of stepping is similar in thinking to a grid-based model, but allows for more realistic stepping directions. The same convergence approach is applied when this equilibrium state is disturbed by external electric-field loading leading to "Equilibrium 2." For this case, the first term in Eq. (1) is no longer zero.

Because a large number of random distributions must be considered, the challenge in this approach is the computational time sometimes required to reach convergence. The current system is considered converged only when all ion steps are rejected. Convergence for a single run is typically reached within 2500 iterations, but considerably more steps are sometimes required. In the parametric studies, 1000 runs of each configuration are considered.

\section{DEFINING THE BASE LINE CASE}

\section{A. Step length}

Various initial step lengths, spanning two orders of magnitude, are considered where all step lengths are normalized with respect to cation radius. The various predictions are compared based on the number of iterations to equilibrium and lowest predicted final energy state (with and without external load).

The starting incremental step sizes range from $2 r_{\text {cat }}$ to $r_{\text {cat }} / 100$. The starting incremental step size is applied throughout the process of establishing Equilibrium 1 from the initialized 26 uniformly distributed anions and 26 randomly distributed cations. Because the average force acting on the ions at the start of this process is significantly larger than that acting on the ions when this equilibrium state is disturbed by an externally applied electric field, the step size is reduced in the second iteration process in proportion to the reduction in initial average force. Application of a simple ratio of forces for reducing step size is justified by the realization that the second action of the model is in fact attempting to use an electrostatic approach to capture a dynamic response where a larger step corresponds to a higher velocity. The significant dynamic effects not explicitly addressed in the foregoing development are viscous drag and electrodynamic effects. The drag force effect in this case is approximately, directly proportional to velocity, ${ }^{27}$ and thus justifies the use of a step length which corresponds to smaller velocities. The redirection of ion movement introduced by electrodynamic effects is rather complex; it is assumed that scaling the step with force will not introduce significant error in this estimation.

It is found that the force acting on an ion in the first iteration is $\sim 2 \times 10^{-11} \mathrm{~N}$, while the force acting on an ion when the subsequent equilibrium is disturbed varies from $\sim 8 \times 10^{-12}$ to $\sim 2 \times 10^{-13} \mathrm{~N}$, where the values are larger for larger initial step sizes. Thus, the factor decreasing the step size in the second stage of the model ranged from $\sim 2$ to $\sim 135$, and subsequently led to significant variation in the number of iterations to Equilibrium 2. Table II shows the predictions for each case; Fig. 3 illustrates the definition of "ion pairs," where center to center distance of the oppositely charged ions must be less than or equal to $r_{\max }$ (Table I).

The step size selected as most appropriate is initialized to a length of $r_{\text {cat }} / 50$ (in Table II). Based on the extent of energy decrease away from the initialized random ion distribution (column $\Delta$ En1), as well as the total energy decrease of the system after it has been perturbed by an external electric field (column $\Delta \mathrm{En} 2$ ), it is clear that convergence is reached at the step size of $r_{\text {cat }} / 40$, however, when the total number of iterations over both loading sequences is considered, application of $r_{\text {cat }} / 50$ will give faster convergence.

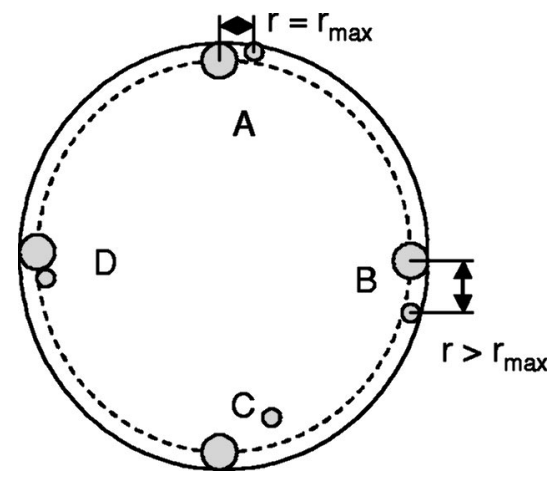

FIG. 3. (a) An ion pair, with an outer cation. (b) An unpaired anion and cation, with an outer cation. (c) An unpaired anion and cation. (d) An ion pair. 
TABLE III. Identification of an alternate cluster surface-tension constant (averages over 10 random distributions, reported for Equilibrium 1).

\begin{tabular}{cccccc}
\hline \hline \multirow{2}{*}{$x \gamma_{b}$} & $x k_{p c}$ & $\begin{array}{c}R_{\text {ave }} \\
(\mathrm{nm})\end{array}$ & $\begin{array}{c}\text { Std Dev } \\
(\mathrm{pm})\end{array}$ & $\begin{array}{c}\text { Outer } \\
\text { cations }\end{array}$ & Pairs \\
\hline \multirow{2}{*}{0.05} & 0.1 & 1.84 & 11.2 & 3.9 & 19.6 \\
& 1 & 1.82 & 17.9 & 3.3 & 19.0 \\
& 10 & 1.86 & 3.58 & 3.2 & 21.2 \\
\multirow{2}{*}{0.1} & 0.1 & 1.85 & 5.50 & 1.0 & 19.9 \\
& 1 & 1.84 & 10.9 & 1.9 & 18.8 \\
\multirow{2}{*}{1} & 0.1 & 1.86 & 0.585 & 8.1 & 20.7 \\
& 1 & 1.86 & 1.43 & 5.8 & 18.9 \\
& 10 & 1.86 & 1.08 & 3.9 & 21.4 \\
10 & 1 & 1.86 & 0.168 & 6.1 & 20.3 \\
\hline \hline
\end{tabular}

Moreover, the cases of $r_{\text {cat }} / 40, r_{\text {cat }} / 50$, and $r_{\text {cat }} / 100$ were also reconsidered over 100 runs; the trends given in Table II remained consistent over 100 runs.

\section{B. Cluster surface-tension coefficient}

The foregoing development of force and energy expressions relies largely on continuum (or macroscale) concepts, and subsequently, the macroscopically determined surfacetension coefficient, $\gamma$, is applied. However, theoretical and computational studies on the length scale of nanometers present an interesting challenge in that it is not truly continuum in nature nor is it quantum. Moreover, this is the length scale studied in molecular dynamics, where quasicontinuum ball-and-spring approaches are typically applied, ${ }^{28,29}$ as has been done in this effort. Recognizing that the constant surface-tension coefficient derives from the intermolecular forces of a pure substance, one should expect that this constant will be locally altered when a single cation or anion group is acting upon the cluster surface. A detailed study of this effect is beyond the scope of this work, however, an indirect assessment is warranted.

It is found that application of the macroscopically determined (base line) value for cluster surface-tension constant, $\gamma_{b}$ leads to "stiff" surface response. Table III provides predicted cluster response to variation in $\gamma$; Fig. 3 illustrates the definition of an outer cation, where the center of the cation must have radial position at least equal to the initialized radial position of the anions. Based on the predictions in Table III, parametric studies are considered for both $\gamma_{b}$ and $0.05 \gamma_{b}$. This alternate value is considered because it is the largest value for which the pendant chain force may have any significant impact on cluster size and response, and thus allows further insight into the role of each of the key parameters (cluster surface, pendant chain, electrostatic, and external field effects).

\section{MODEL VALIDATION AND PREDICTIONS}

The following case studies are investigated for 1000 random distributions: variation of cluster surface tension, nonuniform anion distribution, and variation of initialized cluster size. The latter two cases are considered for both the base line cluster surface-tension constant, $\gamma_{b}$, as noted in Table I, as well as for the reduced cluster surface tension of $0.05 \gamma_{b}$, as noted in Table III. In all cases, anions are initialized to positions on the surface of the cluster with the $r$ coordinate set to $R_{\mathrm{cl}}-R_{\mathrm{an}}$.

For clarity and brevity, predictions for each are provided in graphic form, showing only Equilibrium 1 response. Any variations in response due to the application of external field are described in the text. Each graph considers the number of cations positioned at the outer extremes of the cluster (outer cations), the number of anion-cation pairs ("pairs"), and the average cluster radius $\left(r_{\text {ave }}\right)$.

\section{A. Case 1: Variation of cluster surface tension}

This study of the variation in cluster surface tension repeats three of the cases addressed in Table III, but for 1000 random distributions rather than 10 . This serves two purposes: it gives insight into the statistical validity of the results for each case (Table IV), while simultaneously allowing

TABLE IV. Statistical validity: Comparing the predictions for 10 random samples to those of 1000 random samples for three different cluster surface-tension constants.

\begin{tabular}{|c|c|c|c|c|c|c|}
\hline \multicolumn{3}{|c|}{ (Equilibrium 1/Equilibrium 2) 10 Cases } & \multicolumn{4}{|c|}{ (Equilibrium 1/Equilibrium 2) ${ }_{1000}$ Cases } \\
\hline & \multicolumn{2}{|c|}{$0.1 \gamma_{b}$} & \multicolumn{2}{|c|}{$1.0 \gamma_{b}$} & \multicolumn{2}{|c|}{$10.0 \gamma_{b}$} \\
\hline $\begin{array}{c}\text { Outer } \\
\text { cations }\end{array}$ & $4.4 / 4.4$ & $4.1 / 4.0$ & $5.8 / 5.7$ & $6.1 / 6.0$ & $6.1 / 5.4$ & $6.4 / 5.7$ \\
\hline Std Dev ${ }^{\mathrm{a}}$ & $2.0 / 1.6$ & $1.5 / 1.5$ & $1.5 / 1.3$ & $1.4 / 1.4$ & $1.4 / 1.5$ & $1.5 / 1.3$ \\
\hline Pairs & $19.1 / 19.2$ & $18.6 / 18.6$ & $18.9 / 18.9$ & $19.1 / 19.1$ & $20.3 / 20.3$ & $20.6 / 20.6$ \\
\hline Std Dev ${ }^{\mathrm{a}}$ & $2.0 / 1.8$ & $1.8 / 1.8$ & $2.2 / 2.2$ & $1.8 / 1.8$ & $1.6 / 1.6$ & $1.6 / 1.6$ \\
\hline$R_{\text {ave }}(\mathrm{nm})$ & $1.84 / 1.84$ & $1.84 / 1.84$ & $1.86 / 1.86$ & $1.86 / 1.86$ & $1.86 / 1.86$ & $1.86 / 1.86$ \\
\hline $\begin{array}{l}\text { Std Dev } \\
(\mathrm{pm})\end{array}$ & $11.0 / 10.9$ & $10.9 / 10.8$ & $1.4 / 1.3$ & $1.4 / 1.4$ & $0.9 / 0.2$ & $1.0 / 0.2$ \\
\hline $\begin{array}{l}\text { Std Dev }{ }^{a} \\
(\mathrm{pm})\end{array}$ & $3.7 / 2.7$ & $2.8 / 2.7$ & $0.4 / 0.5$ & $0.3 / 0.3$ & $0.2 / 0.1$ & $0.2 / 0.0$ \\
\hline \multirow[t]{2}{*}{ Iterations } & $1174 /$ & $1320 /$ & $1290 /$ & $1331 /$ & $1303 /$ & $1380 /$ \\
\hline & 1782 & 851 & 999 & 1402 & 5025 & 4028 \\
\hline
\end{tabular}

${ }^{\mathrm{a}}$ Case to case standard deviation.

${ }^{\mathrm{b}}$ Anion to anion standard deviaion. 

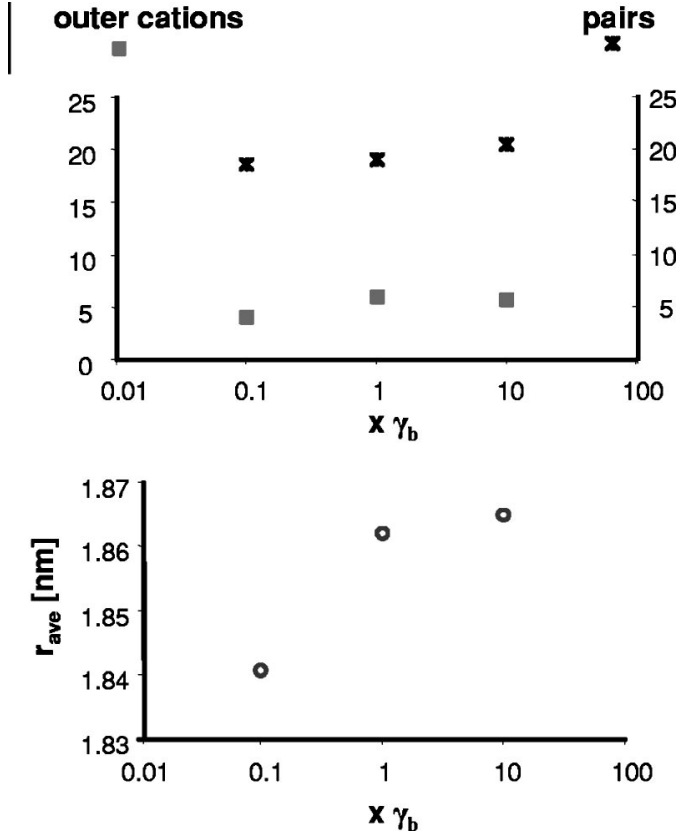

FIG. 4. Variation of cluster surface tension. (a) The predicted number of ion pairs $(1.6<$ standard deviation $<1.8)$ and outer cations $(1.4<$ standard deviation $<1.5$ ). (b) The predicted average cluster radius.

insight into the impact of cluster surface force/energy (Fig. 4).

Referring to Table IV, consider first the issue of statistical validity. For each surface tension considered, cluster size and shape predictions are nearly identical for 10-run and 1000 -run cases. Only the standard deviation of the $r_{\text {ave }}$ values from case to case varies (as opposed to the standard deviation of the $r$ values within each case). For both 10 runs and 1000 runs, the tolerance on the prediction is quite tight, and becomes slightly more so at 1000 runs for most of the noted scenarios; for all scenarios, this standard deviation value does not vary significantly from the 10-run case to the 1000 run case predictions. When considering the average number of iterations, there is significant variation between the anticipated iterations (10 runs) and the average actually realized. However, the difference favors a faster convergence than expected. Lastly, the most significant variations observed between the 10-run case and the 1000-run case studies are the number of outer cations and the number of pairs. However, this variation for 1000 runs as compared to 10 runs is only fractional. Thus, because both the number of cases considered and the variation in a critical material property $(\gamma)$ varied over multiple orders of magnitude in the above study, while the predictions remained effectively constant, the remaining cases will be assumed to be statistically valid. Moreover, the standard deviation of the $r_{\text {ave }}$ values within each case is consistently two or more orders of magnitude smaller than the prediction itself; this trend persisted through the study, and is thus omitted from further reporting.

The above discussion of the tight tolerance (small standard deviations) in the predictions also addresses the issue of convergence to local energy minima. Specifically, cluster convergence within an ionomer should not be expected to achieve an absolute minimum energy state because of the
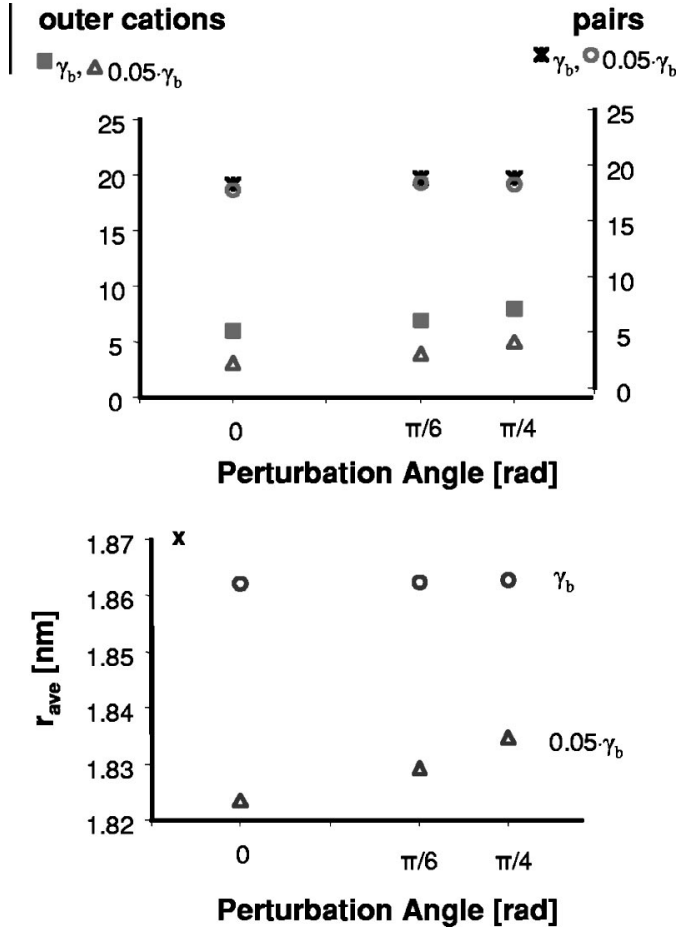

FIG. 5. Ion position perturbation. (a) The predicted number of ion pairs $(1.4<$ standard deviation $<1.7)$ and outer cations $\quad(1.8<$ standard deviation $<2.2$ ). Zero pairs and zero outer cations are predicted for uniform anion and cation-initialized state. (b) The predicted average cluster radius. $X$ corresponds to the prediction for uniform anion and cation-initialized state.

dynamic nature of fluid/ion morphology. Thus, typical energy minima, though not necessarily an absolute minimum, should be expected as the norm; the above predictions indicate that this norm falls within a rather tight range.

Next, consider the predictions themselves (Fig. 4). For the two stiffest cases, despite significant ion motion, the final cluster size is virtually identical to the initialized size. There is also an increase in the predicted number of pairs and outer cations as $\gamma$ increases. Lastly, in all but the predicted number of outer cations for the stiffest $\gamma$, there is no discernable difference between Equilibriums 1 and 2. For the exception case, the predicted number of cations positioned at the outer region of the cluster drops from 6.4 to 5.7 ions per cluster upon the application of an electric field. When the ions with positive and negative $z$ components are considered separately, it is found that about $2 / 3$ of this reduction occurs in cations positioned in the negative $z$ direction, and thus the motion is in the direction of the applied field. Lastly, the magnitude of the internally generated electric field is on the order of $10^{8} \mathrm{~V} / \mathrm{m}$ for all cases.

\section{B. Case 2: Variation of anion/cation-initialized positions $-\gamma_{b}$}

This study (Fig. 5) considers the effect of the ion distribution. Perturbing the position of the anions away from the uniformly distributed case is considered first, followed by consideration of uniform distribution of both the cations and anions. For the base line case $\left(\alpha_{\text {per }}=0\right)$, the internally generated electric fields are on the order of $10^{8} \mathrm{~V} / \mathrm{m}$; for both of the perturbed cases, these fields are on the order of $10^{7} \mathrm{~V} / \mathrm{m}$. 


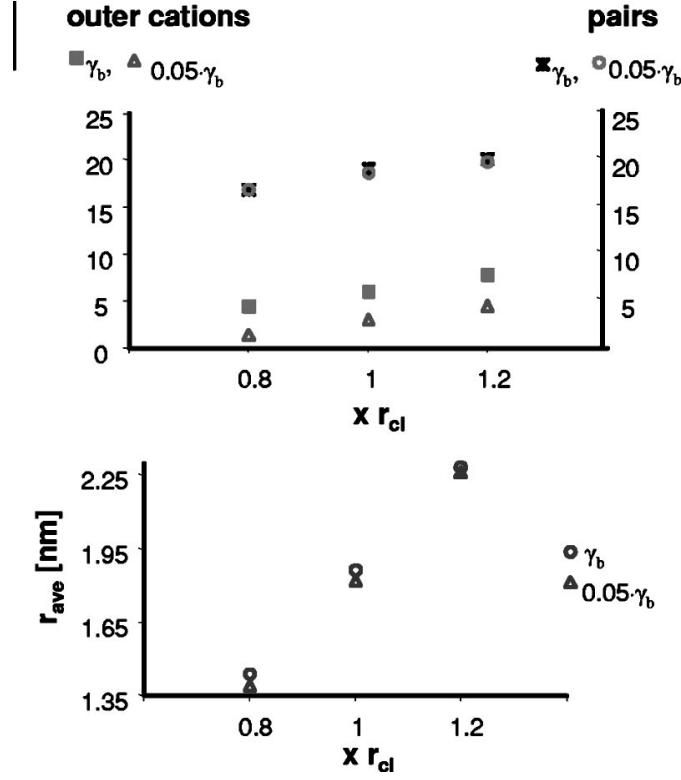

FIG. 6. Initialized cluster radius. (a) The predicted number of ion pairs $(1.4<$ standard deviation $<2.5)$ and outer cations $\quad(1.1<$ standard deviation $<1.7$ ). (b) The predicted average cluster radius.

For the case where both the anions and cations are uniformly and radially distributed as pairs on the surface, the fields are on the order of $10^{8} \mathrm{~V} / \mathrm{m}$. As in previous cases, no discernable changes in the systems are identified upon application of the external electric field.

\section{Case 3: Variation of anion/cation-initialized positions $-0.05 \gamma_{b}$}

This study (Fig. 5) again considers the impact of uniform versus random anion distribution, but for the case of $0.05 \gamma_{b}$. The internally generated electric fields of the perturbed cases are on the order of $10^{7} \mathrm{~V} / \mathrm{m}$. For the case where both the anions and cations are uniformly distributed as pairs on the surface, the fields are on the order of $10^{8} \mathrm{~V} / \mathrm{m}$. No discernable change in the system is identified upon application of the external electric field in any of the cases.

\section{Case 4: Variation of initialized cluster size- $\gamma_{b}$}

Referring to Fig. 6, in all cases there is a tendency for the cluster to reach an equilibrium size which is tenths of percent smaller than its initialized size. For the largest initialized case, the predicted number of outer cations upon application of an external electric field decreases (Equilibrium 2). It is found that there is an even distribution of cations moving inward on both sides of the cluster.

\section{E. Case 5: Variation of initialized cluster size $-0.05 \gamma_{b}$}

The impact of the assumed initial cluster size for $0.05 \gamma_{b}$ is considered in Fig. 6. There is again a tendency for the cluster to reach an equilibrium size which is smaller than its initialized size, but with decreases ranging from $1.5 \%$ to $3.5 \%$; these values constitute nontrivial strain response. Again, there is a decrease in the predicted number of outer cations for the largest initialized radius at Equilibrium 2; the numbers of cations are evenly distributed to the upper and lower portions of the cluster.

\section{DISCUSSION}

Because a significant number of case studies are considered, a corresponding number of issues warrant discussion. Thus, this discussion will skip the bulk of the "intuitive" predictions, such as reduced anion movement in response to increased cluster surface tension. The discussion is thus limited to general tendencies, to significant case by case observations, and to the less, or sometimes counterintuitive predictions.

\section{A. General tendencies}

The first significant observation from the model predictions is the pervading tendency for an incomplete ion pairing, while simultaneously, a nontrivial number of cations are predicted to assume equilibrium positions at the outer extremities of the cluster. When taken together, this suggests that cluster equilibrium states are predisposed to cation transport. This is consistent with the transport mechanism proposed by Datye et al., ${ }^{12}$ where the nontrivial number of pairs at the cluster surface still provides the impetus for transport, but with the added understanding of how it is possible that free cations are available for transport. Further, the number of predicted pairs is relatively insensitive to variations in cluster parameters, where the predicted number of pairs is typically between 18.6 and 20.6 (excluding initialized radius effects). It is believed that the prediction of incomplete pairing is related to (i) the inclusion of the Pauli repulsion effect addressed in the electrostatic interaction [Eq. (6)], (ii) restricted anion mobility, and (iii) applying an initialized, nonuniform cation distribution. The last point addresses the impossibility of uniformly introducing new cations to individual clusters within the material in the fabrication stage. If this prediction is considered in conjunction with the global IPMC modeling developments of Nemat-Nasser and $\mathrm{Li}^{17,18,30}$ where the assumption of uniform distribution of ion pairs is applied in the development of an expression for the internal cluster pressure and corresponding effects on predicted stiffness and boundary layer thickness, their model would tend to underpredict the actuation response. Application of the results of the current effort could easily be introduced to these global models in order to better account for this more realistic cluster state.

The number of cations predicted at the outer extremes of the cluster is sensitive to variations in cluster parameters, with a low prediction of 3.1 and a high of 8.2. It is presumed in this effort that a large number of cations positioned at the outer positions corresponds to a state that is transport facilitated. The rationale is twofold. First, an outer cation is positioned favorably for transport to a neighboring cluster. Second, in the event that cation is inhibited from transport by pairing, by virtue of establishing a pair, it opens a neighboring vicinity for other cations to transport with minimal interference. Both the perturbation of anion positioning and variation of the cluster surface-tension constant impact the 
predicted number of outer cations. In general, a stiffer $\gamma$ leads to increased cations at the surface. The value in this prediction is that by varying either the solvent introduced or the cation (and thereby altering $\gamma$ ), control over this parameter is envisioned. The challenge here is that introduction of new solvents or alternate cations will necessarily impact the self-organized cluster size and shape, over which there is little control at present; as explored in Cases 4 and 5, initialized cluster size has dramatic impact on model predictions. Thus, to establish reliable control over the number of cations positioned at the outer extremities of the cluster, and facilitate transport, an optimization of various fluid/polymer interactions would be required.

One of the goals of this effort is to investigate the impact of the classically assumed spherical cluster shape. In the studies considered here, the standard deviation in position from anion to anion is two orders of magnitude lower than the prediction itself, where anion position is assumed to dictate cluster size and shape. Thus, for an initialized spherical shape, even under significant ion motion, the shape remains essentially spherical.

Some of the cases have predictions of reduced internally generated electric fields. Because the result is unexpected, it is reported. However, because these calculations are done only at six specific points in space (at $x, y$, and $z= \pm R_{\mathrm{cl}}$ ), it is possible that the prediction is not general enough to be treated as a global prediction. It is, however, conceivable that significantly different electric fields will be generated at points exterior to the cluster based on the cluster state.

With respect to the application of an external electric field, where the magnitude of the applied field is consistent with average fields applied in IPMC applications, no significant variation in cluster state is predicted. There are multiple possible explanations for this. First, the model is fundamentally electrostatic in nature, while response to electric field is fundamentally dynamic. While an attempt to address this issue is made via the reduction of step length upon application of an electric field, it is conceivable that this served only to "fine tune" the low-energy state. This is suggested by the predictions of decreasing numbers of outer cations upon the application of an external electric field in Cases 1, 4, and 5. Another possible consideration is that cation transport should be expected, where the current model offers no transport paths to neighboring clusters, while their existence is implicitly assumed in allowing the clusters to expand/contract. Lastly, it is possible that the applied electric field is simply too low in magnitude to affect any significant response; as noted in an earlier work, ${ }^{19}$ the electrode structure of IPMCs is dendritic in nature and will necessarily result in locally high electric fields. Thus, no conclusion may be drawn with respect to electric-field loading based on the foregoing studies.

\section{B. Initialized cluster size}

As explored in Cases 4 and 5, initialized cluster size has significant impact on model predictions. Selection of the initialized cluster size must be carefully considered because this state is the only state where cluster surface and pendant chain effects are zero. This is a necessary condition, as it is understood that in an equilibrium configuration, each of these energy terms will be nonzero and mutually balanced. Because it is impossible to anticipate where that balance will occur, some initial state must be assumed. The base line cluster radius applied is based on Nafion ${ }^{\circledR}$ fully solvated with water and sodium as the cation, as estimated from the works by Datye et al. ${ }^{12}$ When the base line cluster surface tension, $\gamma_{b}$, is also employed, the equilibrium radius is essentially unchanged. However, when a reduced cluster surface-tension constant is employed, $0.05 \gamma_{b}$, significant contraction is predicted, even when the initialized size is itself reduced. Because the base line cluster surface-tension constant, $\gamma_{b}$, is believed to be too large at this length scale, it is proposed that in order to use this type of model for a specific case study (as opposed to the trend study in the current effort), it would need to be fitted to the appropriate cluster radius. In other words, a guess method for the initialized state would have to be employed until a realistic equilibrium cluster size is predicted; corresponding pairing and outer cation predictions could then be used to anticipate the extent to which the configuration is transport facilitated. Thus, this parameter must be an informed value (preferably identified experimentally) for the specific case of interest.

Another interesting prediction arising from this study is the decreased number of outer cations and pairs predicted as the initialized cluster radius is reduced. One might expect each of these values to increase under the conditions of "close quarters." What is likely happening is that the ions are finding uniform distributions to minimize the energy state (in response to Pauli repulsion) rather than pairing or moving to outer positions.

\section{Initialized ion distribution}

One of the goals of this effort is to address the impact of the classically assumed, uniform distribution of anions along the cluster surface and perfect corresponding alignment of cations, forming nested ion spheres. To highlight the significant difference between this modeling approach and those that assume this uniform distribution, a perfectly uniform case as described is also tested in Cases 2 and 3. As expected, when the perfectly uniform case is employed as the initialized state, the system change is negligible from the initialized state. However, as previously noted, the incomplete ion pairing and outer cations predicted by the various random distributions should in fact facilitate transport; thus, again, the assumption of uniform distribution may tend to underpredict this material response. Moreover, the predicted number of ion pairs formed is approximately constant over the variations in perturbation, while the predicted number of outer cations increases; thus, it is presumed that transport is predicted to be facilitated by the more realistic random ion placement, though it may be somewhat more challenging to incorporate this prediction into the global IPMC models noted.

Another interesting prediction of this study is the modest increase in cluster size as perturbation increases (though still smaller than the predicted size for the perfectly uniform 
case). This is likely the result of the electrostatic effects encouraging the aforementioned uniform distribution, while the pendant chain resistance to radial distortion is now somewhat increased due to the added tangential displacements.

\section{Cluster surface stiffness}

Case 1 most directly addresses variation of cluster surface stiffness. It is to be expected that variation of the cation will affect the cluster surface tension, which in turn is known to affect clustering; this is supported by the well-documented variation in water uptake and cluster morphology with variation of the introduced cation. Also, noting that the results of the current effort emphasize the significant impact cluster morphology has on whether a cluster is transport facilitated, it is concluded that the cluster surface tension plays a significant, if not sometimes dominant, role in cluster morphology for varying levels of water uptake, which in turn plays a significant role in facilitating the cation transport leading to actuation response. A detailed, case by case study is necessary to assess the exact impact of this parameter for variations in cation, water uptake, or other solvent uptake.

\section{CONCLUSIONS}

In this work the impact of assuming spherical cluster shapes with uniform ion distribution is investigated. The model predicts that, due to inhibited anion motion via elastic and surface energy effects, combined with Pauli repulsion, ion paring is rarely complete, thus facilitating cation transport. Overall, this model suggests that application of the assumption of uniform charge distribution over a spherical cluster shape in global material models will tend to underpredict the observed macroscopic actuation response; in general, these predictions are easily incorporated into the global models. The spherical cluster shape is essentially maintained throughout the various case studies. Further, this study introduces the significant role of cluster surface tension in ultimate actuation response and thus represents a potential mechanism for control over transport properties, and subsequently, over actuation response.

\section{ACKNOWLEDGMENT}

The authors would like to acknowledge the support of the National Science Foundation Grant No. CMS-0093889, from which publications charges for article have been paid in part.
${ }^{1}$ K. Sadeghipour, R. Salomon, and S. Neogi, Smart Mater. Struct. 1, 172 (1992).

${ }^{2}$ K. Oguro, Y. Kawami, and H. Takenaka, Osaka Kogyo Gijutsu Shikensho Hokoku 43, 21 (1992)

${ }^{3}$ Y. Osada and M. Hasebe, Chem. Lett. 1285 (1985).

${ }^{4}$ M. Irie, Macromolecules 19, 2890 (1986)

${ }^{5}$ T. Shiga and T. Kurauchi, J. Appl. Polym. Sci. 39, 2305 (1990).

${ }^{6}$ M. Shahinpoor, Smart Mater. Struct. 1, 91 (1992).

${ }^{7}$ M. Shahinpoor, Y. Bar-Cohen, J. O. Simpson, and J. Smith, Smart Mater. Struct. 7, R15 (1998).

${ }^{8}$ M. Mojarrad and M. Shahinpoor, Proc. SPIE 2779, 1012 (1996).

${ }^{9}$ S. Nemat-Nasser, J. Appl. Phys. 92, 2899 (2002).

${ }^{10} \mathrm{~K}$. Farinholt, K. Newbury, M. Bennet, and D. Leo, "An Investigation into the Relationship between Charge and Strain in Ionic Polymer Sensors," First World Congress on Biomimetics and Artificial Muscles, Albuquerque, NM, 9-11 December 2002.

${ }^{11}$ W. Y. Hsu and T. D. Gierke, Macromolecules 15, 101 (1982).

${ }^{12}$ V. K. Datye, P. L. Taylor, and A. J. Hopfinger, Macromolecules 17, 1704 (1984).

${ }^{13}$ V. K. Datye and P. L. Taylor, Macromolecules 18, 1479 (1985).

${ }^{14}$ A. Lehmani, S. Durand-Vidal, and P. Turq, J. Appl. Polym. Sci. 68, 503 (1998).

${ }^{15}$ P. G. de Gennes, K. Okumura, M. Shahinpoor, and K. J. Kim, Europhys. Lett. 50, 513 (2000).

${ }^{16}$ Electroactive Polymer (EAP) Actuators as Artificial Muscles-Reality, Potential, and Challenges, edited by Y. Bar-Cohen (SPIE-The International Society for Optical Engineering, Bellingham, Washington, 2001).

${ }^{17}$ J. Y. Li and S. Nemat-Nasser, Mech. Mater. 32, 303 (2000).

${ }^{18}$ S. Nemat-Nasser and J. Y. Li, J. Appl. Phys. 87, 3321 (2002).

${ }^{19}$ L. Mauck Weiland and D. J. Leo, Smart Mater. Struct. 13, 323 (2004).

${ }^{20}$ J. A. Stratton, Electromagnetic Theory (McGraw-Hill, New York, 1941).

${ }^{21}$ K. M. Newbury and D. J. Leo, J. Intell. Mater. Syst. Struct. 14, 343 (2003);

${ }^{22}$ A. L. Rouff, Introduction to Materials Science (Robert E. Krieger, Huntington, NY, 1979).

${ }^{23}$ L. H. Van Vlack, Elements of Materials Science and Engineering, 5th ed. (Addison-Wesley, Reading, MA, 1985).

${ }^{24}$ L. D. Landau and E. M. Lifshitz, Fluid Mechanics, 2nd ed. Course of Theoretical Physics, Vol. 6 (Butterworth-Heinemann, Linacre House, Oxford, 1987)

${ }^{25}$ R. B. Dooley, Release on Surface Tension of Ordinary Water Substance (Electric Power Research Institute, Palo Alto, CA, 1994).

${ }^{26}$ R. B. Dooley Release on the Static Dielectric Constant of Ordinary Water Substance for Temperatures from $238 \mathrm{~K}$ to $873 \mathrm{~K}$ and Pressures up to $1000 \mathrm{MPa}$ (Electric Power Research Institute, Palo Alto, CA, 1997).

${ }^{27}$ J. N. Murrell and A. D. Jenkins, Properties of Liquids and Solutions, 2nd ed. (Wiley, New York, 1994).

${ }^{28}$ R. A. Serway, Physics: For Scientists and Engineers (Saunders College, New York, 1982).

${ }^{29}$ M. Fermeglia, S. Pricl, and G. Longo, Chem. Biochem. Eng. Q. 17, 19 (2003).

${ }^{30}$ K. Kremer and F. Müller-Plathe, MRS Bull. 26, 205 (2001).

${ }^{31}$ S. Nemat-Nasser, J. Appl. Phys. 92, 2899 (2002). 